\title{
Grassmannian heterotic sigma model
}

\author{
Michael Kreshchuk, ${ }^{1,2}$ Evgeniy Kurianovych, ${ }^{2}$ and Mikhail Shifman ${ }^{2,3}$ \\ ${ }^{1}$ Department of Physics, Tufts University, Medford, Massachusetts 02155, USA \\ ${ }^{2}$ School of Physics and Astronomy, University of Minnesota, Minneapolis, Minnesota 55455, USA \\ ${ }^{3}$ William I. Fine Theoretical Physics Institute, University of Minnesota, \\ Minneapolis, Minnesota 55455, USA
}

(Received 10 February 2019; published 11 June 2019)

\begin{abstract}
We study the nonminimal supersymmetric heterotically deformed $\mathcal{N}=(0,2)$ sigma model with the Grassmannian target space $\mathcal{G}_{M, N}$. To develop the appropriate superfield formalism, we begin with a simplified model with flat target space, find its beta function up to two loops, and prove a nonrenormalization theorem. Then we generalize the results to the full model with the Grassmannian target space. Using the geometric formulation, we calculate the beta functions and discuss the 't Hooft and Veneziano limits.
\end{abstract}

DOI: 10.1103/PhysRevD.99.125005

\section{INTRODUCTION}

Sigma models have a long history dating to 1960 [1]. In 1975, Polyakov [2] was the first to observe that the $\mathbb{O}(3)$ sigma model is asymptotically free and provides a laboratory for modeling four-dimensional gauge theories, allowing one to study similar phenomena in a simplified setting. Heterotically deformed supersymmetric models are of particular interest due to their close connection to the world-sheet theories on vortex strings supported in the $\mathcal{N}=1$ Super-Yang-Mills theories in four dimensions [3]. Additionally, such models are also interesting from the mathematical viewpoint [4-9]. In recent years heterotic sigma models have attracted significant attention [10-28] (for a review see [29]).

The most widely considered target spaces of the sigma models studied in the literature are $\mathbb{S}^{N}=\mathbb{S O}(N+1) /$ $\mathbb{S O}(N)$ [2] and $\mathbb{C P}(N)=\mathbb{S U}(N+1) / \mathbb{S U}(N) \times \mathbb{U}(1)$ [30-32]. Their supersymmetric versions were introduced in [32] and [33-35], respectively. These arise naturally as the world-sheet effective low-energy theories of the nonAbelian string solutions. In this paper, we turn to the generalization of $\mathbb{C P}(N)$, the Grassmannian target space [36-39],

$$
\mathcal{G}_{M, N}=\frac{\mathbb{S U}(N+M)}{\mathbb{S U}(N) \times \mathbb{S U}(M) \times \mathbb{U}(1)} .
$$

Published by the American Physical Society under the terms of the Creative Commons Attribution 4.0 International license. Further distribution of this work must maintain attribution to the author(s) and the published article's title, journal citation, and DOI. Funded by SCOAP ${ }^{3}$.
Since the Grassmannian is a Kähler manifold, the Lagrangian of the $(2,2)$ model can be written as

$$
\mathcal{L}_{(2,2)}=\int d^{4} \theta K\left(\Phi, \Phi^{\dagger}\right)
$$

where $K\left(\Phi, \Phi^{\dagger}\right)$ is the Kähler potential. In our paper, we consider the so-called nonminimal model in which supersymmetry is broken down to $(0,2)$ by the addition of an extra deformation term to the Lagrangian (2). In this way, one ends up with two types of interactions and two corresponding coupling constants. The interaction of the first type is owing to geometry of the target space, while the interaction of the second type is due to the heterotic deformation.

In Sec. I, we develop the superfield formalism and study the limit of the heterotic deformation assumed to be much stronger than the interaction due to the target-space geometry. To this end, we consider the simplified flat target-space model, a straightforward generalization of the case considered in [18]. In Sec. II, we prove a nonrenormalization theorem for this model. In Sec. III, we consider the full model, with both the curved target space and the heterotic deformation. There are two ways of analyzing interactions induced by the target-space constraint: (a) by approaching the curved geometry directly, and (b) by introducing extra gauge fields. In the current work we rely on the former approach. For its implementation, several basic tools from the geometry of the Grassmannian are required. A brief review of this machinery is also provided in Sec. III. In Sec. IV, we write down the beta functions of the full model, while their 't Hooft and Veneziano limits are discussed in Sec. V. Our notation, as well as the background field method, is reviewed in the appendixes. 


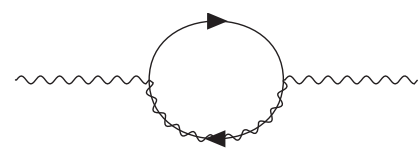

(a)

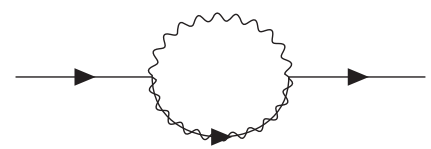

(b)

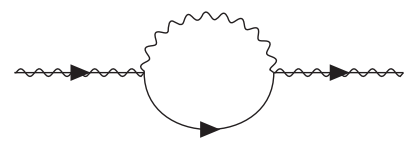

(c)

FIG. 1. One-loop corrections. The wavy line is $A$, the solid line is $B$, and the composition of the wavy and solid lines is $\mathcal{B}$.

\section{FLAT TARGET-SPACE MODEL}

The nonminimal heterotic $(0,2)$ model combines the original $(2,2)$ model and its deformation that partly breaks supersymmetry. Before exploring their interplay, let us take a separate look at the two parts-the interaction imposed by geometry and the interaction generated by deformation. The case of the undeformed $(2,2)$ model is well studied in the literature [38-41]. In this section we start by exploring the opposite limit-the heterotically deformed flat target-space model. Since its target space is just the complex linear $\mathbb{C}^{M N}$, we call this model linearized. It is a generalization of the case considered in [18], and is used to develop the appropriate $(0,2)$ formalism. This theory corresponds to the limit of the vanishing target-space curvature of the full model, or, in other words, to the interaction caused by the heterotic deformation much stronger than the interaction arising from geometry. For this linearized model with a trivial metric, we study the heterotic deformation and discuss the running of the coupling constant. Then, we generalize the results to the full model (29).

The complex dimension of the Grassmannian manifold is $M N$. Accordingly, we introduce $M N$ chiral superfields labeled by two indices,

$\Phi^{i \alpha}=\phi^{i \alpha}+\sqrt{2} \theta \psi^{i \alpha}+\theta^{2} F^{i \alpha}, \quad n=1 \ldots N, \quad \alpha=1 \ldots M$.

In terms of the $(0,2)$ superfields, $\Phi^{i, \alpha}$ can be written as

$$
\begin{gathered}
\Phi^{i \alpha}\left(x_{R}+2 i \theta_{R}^{\dagger} \theta_{R}, x_{L}-2 i \theta_{L}^{\dagger} \theta_{L}, \theta_{R}, \theta_{L}\right) \\
=A^{i \alpha}\left(x_{R}+2 i \theta_{R}^{\dagger} \theta_{R}, x_{L}-2 i \theta_{L}^{\dagger} \theta_{L}, \theta_{R}\right) \\
\quad+\sqrt{2} \theta_{L} B^{i \alpha}\left(x_{R}+2 i \theta_{R}^{\dagger} \theta_{R}, x_{L}, \theta_{R}\right) .
\end{gathered}
$$

The superfield $A^{i \alpha}$ represents the chiral supermultiplet; on mass shell it consists of the scalar field and the left-moving fermion,

$A^{i \alpha}=\phi^{i \alpha}\left(x_{R}+2 i \theta_{R}^{\dagger} \theta_{R}, x_{L}\right)+\sqrt{2} \theta_{R} \psi_{L}^{i \alpha}\left(x_{R}+2 i \theta_{R}^{\dagger} \theta_{R}, x_{L}\right)$,

where

$$
x_{L}=\frac{1}{2}\left(x^{0}+x^{1}\right), \quad x_{R}=\frac{1}{2}\left(x^{0}-x^{1}\right) .
$$

The field $B^{i \alpha}$ describes the Fermi supermultiplet that on mass shell contains only the right-moving fermion $\left(F^{i \alpha}\right.$ is an auxiliary field),

$B^{i \alpha}=\psi_{R}^{i \alpha}\left(x_{R}+2 i \theta_{R}^{\dagger} \theta_{R}, x_{L}\right)+\sqrt{2} \theta_{R} F_{\psi}^{i \alpha}\left(x_{R}+2 i \theta_{R}^{\dagger} \theta_{R}, x_{L}\right)$.

To break supersymmetry down to $(0,2)$, we introduce another supermultiplet $\mathcal{B}$,

$\mathcal{B}=\zeta_{R}\left(x_{R}+2 i \theta_{R}^{\dagger} \theta_{R}, x_{L}\right)+\sqrt{2} \theta_{R} F_{\zeta}\left(x_{R}+2 i \theta_{R}^{\dagger} \theta_{R}, x_{L}\right)$.

It has no target-space indices.

In the $(0,2)$ formalism, the Lagrangian of the linearized model acquires the form of ${ }^{1}$

$$
\begin{aligned}
\mathcal{L}_{\text {flat }}= & \frac{1}{2} \int d^{2} \theta_{R}\left[\frac{1}{2} Z_{A}\left(i A^{\dagger}{ }_{i \alpha} \partial_{R} A^{i \alpha}\right)+Z_{B} B^{\dagger}{ }_{i \alpha} B^{i \alpha}\right. \\
& \left.+Z_{\mathcal{B}} \mathcal{B}^{\dagger} \mathcal{B}-Z_{\gamma}\left(\gamma \mathcal{B} B^{i \alpha} A^{\dagger}{ }_{i \alpha}+\text { H.c. }\right)\right] .
\end{aligned}
$$

Here we have already introduced the $Z$-factors. The last term is the one breaking the supersymmetry down to $(0,2)$. We see that such a model corresponds to $M N$ copies of the linearized $\mathbb{C P}(1)$ considered in [18], with $A$ and $B$ fields interacting only via the deformation field.

After eliminating the auxilary fields $F_{\psi}^{i \alpha}$ and $F_{\zeta}$, Eq. (9) reads in components as

$$
\begin{aligned}
\mathcal{L}_{\text {flat }}= & \partial^{\mu} \phi_{i \alpha}^{\dagger} \partial_{\mu} \phi^{i \alpha}+i \bar{\psi}_{i \alpha} \not \partial \psi^{i \alpha}+i \zeta_{R}^{\dagger} \partial_{L} \zeta_{R} \\
& +\left(\gamma \zeta_{R} \psi_{R}^{i \alpha} \partial_{L} \phi_{i \alpha}^{\dagger}+\text { H.c. }\right) \\
& +\gamma^{2}\left(\zeta_{R}^{\dagger} \zeta_{R}\right)\left(\psi_{L i \alpha}^{\dagger} \psi_{L}^{i \alpha}\right)+\gamma^{2}\left(\psi_{R i \alpha}^{\dagger} \psi_{R}^{i \alpha}\right)\left(\psi_{L i \alpha}^{\dagger} \psi_{L}^{i \alpha}\right) .
\end{aligned}
$$

The diagrams for one-loop wave function renormalization are shown in Fig. 1, for two loop in Figs. 2(a) and 2(b) (we don't need them now due to the nonrenormalization of $A$, but they will be used later in the full model), Figs. 3(a) and 3(b) and Figs. 4(a) and 4(b). They give

\footnotetext{
${ }^{1}$ In this section, due to the flatness of the target space, the position of the fields' indices is not of importance. For the same reason, here we do not use the barred indices either.
} 


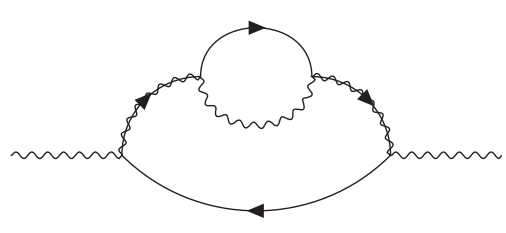

(a)

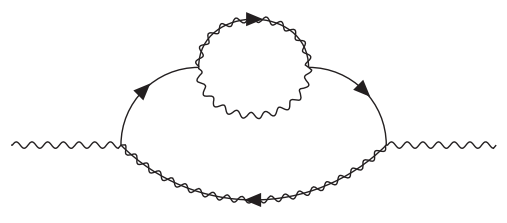

(b)

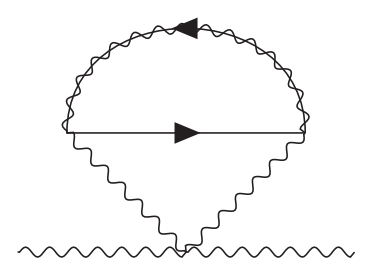

(c)

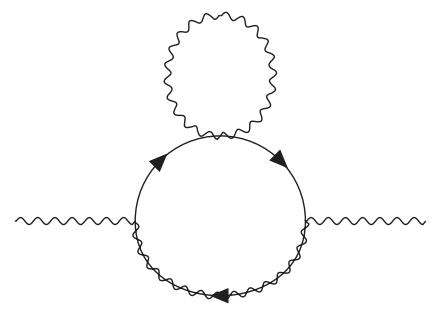

(d)

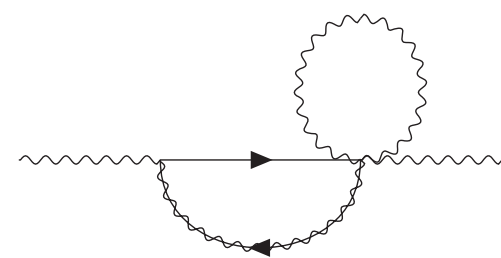

(e)

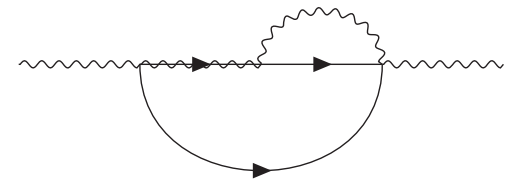

(f)

FIG. 2. Two-loop corrections for A. The diagrams (a) and (b) are common for both the linearized and the full models, while the remaining ones involve vertices with more than three lines that appear only in the geometric model.

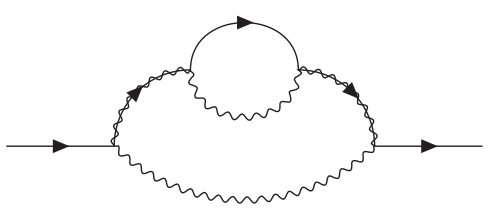

(a)

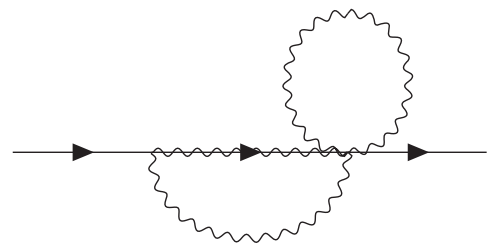

(d)

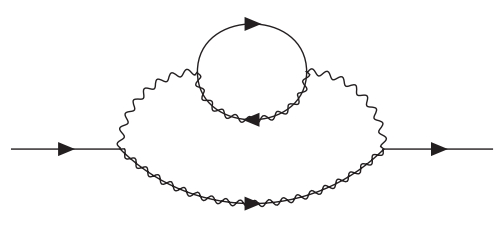

(b)

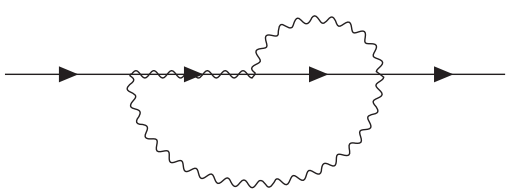

(e)

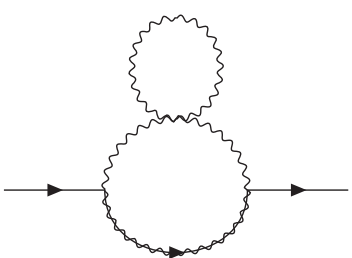

(c)

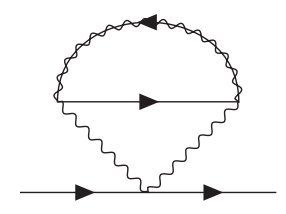

(f)

FIG. 3. Two-loop corrections for $B$. In the same way as above the first two diagrams are applicable for both the linearized and the full models, while the other diagrams appear only in the latter one.

$$
\begin{gathered}
Z_{B}=1+i \gamma^{2} I+\frac{1}{2} M N \gamma^{4} I^{2}, \\
Z_{\mathcal{B}}=1+i M N \gamma^{2} I+\frac{1}{2} M N \gamma^{4} I^{2},
\end{gathered}
$$

where

$$
\begin{aligned}
I & =\int \frac{d^{2-\epsilon} p}{(2 \pi)^{2-\epsilon}} \frac{1}{p^{2}-\mu^{2}}=-\frac{i}{2 \pi \epsilon}+O\left(\epsilon^{0}\right) \\
& =-\frac{i}{2 \pi} \log \left(\frac{M_{\mathrm{uv}}}{\mu}\right) .
\end{aligned}
$$

At the one-loop level, there are no diagrams contributing to renormalization of $\gamma$, and so the beta function is determined solely by the $Z$ factors (11). Consequently, the two twoloop beta function for $\gamma^{2}$ is

$$
\beta\left(\gamma^{2}\right) \frac{\partial \gamma^{2}}{\partial \log \mu^{2}}=\frac{(M N+1) \gamma^{4}}{2 \pi}
$$

As was shown in [18], not only is the beta function for the coupling $\gamma$ exact to all orders in perturbation theory; it does not receive nonperturbative corrections either. The superfield $A$ is also not renormalized due to the theorem proven in [18].

The positivity of the beta function (13) implies the existence of the Landau pole, which indicates that the model should be considered as an effective low-energy theory having some UV completion. We shortly see that 


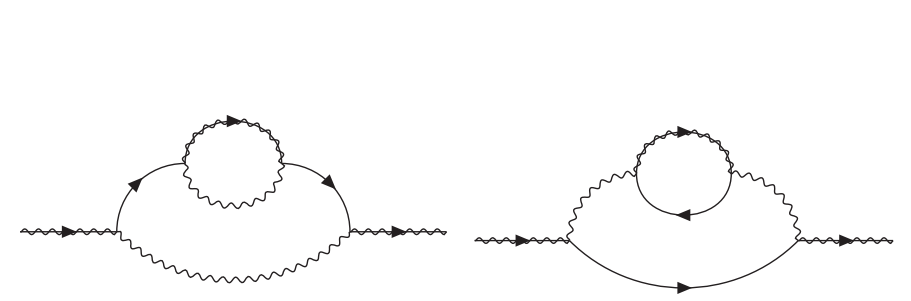

(a)

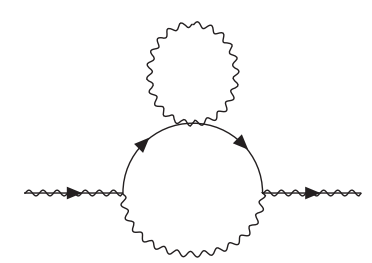

(d) (b)

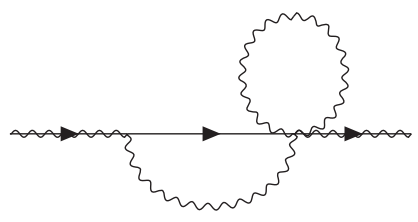

(e)

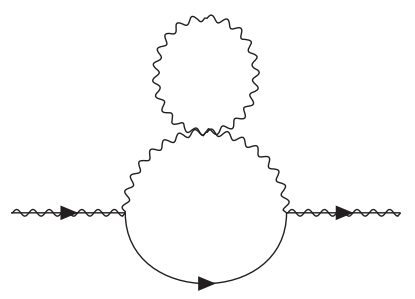

(c)

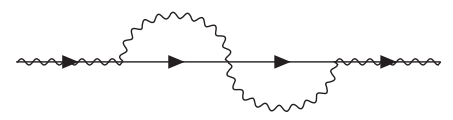

(f)

FIG. 4. Two-loop corrections for $\mathcal{B}$. Once again, the diagrams (a) and (b) are common for both models while the diagrams (c)-(f) are applicable only in the full model.

under certain conditions the full model exhibits the same type of behavior.

\section{NONRENORMALIZATION}

We now prove a version of the nonrenormalization theorem for the interaction term and the kinetic term of the $A$ field. To this end, we use the symmetries and analytic properties analogous to those from [18], and follow the way of reasoning similar to that in [42]. Our theorem is to be valid to all orders in the perturbation theory and, most importantly, nonperturbatively as well.

Let us take a look at the $R$-symmetry. Most of the terms in the Lagrangian are neutral combinations of the type $A^{\dagger} A$, $B^{\dagger} B$, and $\mathcal{B}^{\dagger} \mathcal{B}$. The only term we have to care about is

$$
\gamma \mathcal{B B} A^{\dagger} \text {. }
$$

For this term, we are free to choose three independent $\mathbb{U}(1)$ phases, while the fourth one is defined by the condition of the overall neutrality. So, we have a three-dimensional space whose sample basis is provided in Table I.

Assume that we have a function of $\gamma \mathcal{B B} A^{\dagger}$ and $A^{\dagger} A$. The most general function of the neutral combinations can be expressed as

$$
f\left(\frac{\gamma \mathcal{B} B}{A}, \gamma \mathcal{B} B A^{\dagger}\right)
$$

TABLE I. $\mathbb{U}(1)$ charges of the model.

\begin{tabular}{lccc}
\hline \hline & $\mathbb{U}(1)_{1}$ & $\mathbb{U}(1)_{2}$ & $\mathbb{U}(1)_{3}$ \\
\hline$A$ & 1 & 1 & 1 \\
$B$ & 1 & 0 & 0 \\
$\mathcal{B}$ & 0 & 1 & 0 \\
$\gamma$ & 0 & 0 & 1 \\
\hline \hline
\end{tabular}

The integral over $d^{2} \theta_{R}$ must be invariant under the linear shifts

$$
A^{\dagger} \rightarrow A^{\dagger}+a^{\dagger},
$$

which requires the integrand to be a combination of holomorphic and antiholomorphic functions. Its Laurent expansion in powers of $\gamma \mathcal{B B A} A^{\dagger}$ is exhausted by the constant and linear terms,

$$
f=f_{0}\left(\frac{\gamma \mathcal{B} B}{A}\right)+f_{1}\left(\frac{\gamma \mathcal{B} B}{A}\right) \gamma \mathcal{B} B A^{\dagger} .
$$

At $\gamma=0$ the theory is free, so there should be no negative powers of $\gamma$ in the Laurent series for $f_{0,1}$. $A$ in the denominator and the shift symmetry (16) restrict the positive powers, so $f_{0,1}$ should be constants. We see that there is no dependence on $A^{\dagger} A$; in the same way we can prove the absence of $|B|^{2},|\mathcal{B}|^{2}$, and $|\gamma|^{2}$. After the integration over $d^{2} \theta_{R}, f_{0}$ vanishes,

$$
\int d^{2} \theta_{R} f_{0}=0
$$

Since $f_{1}$, being a constant, does not depend on $\gamma$, it has to be the coefficient from the classical Lagrangian (9).

The renormalized kinetic term for $A$ will look like

$$
F A^{\dagger} \partial_{R} A+F^{\dagger} A \partial_{R} A^{\dagger}
$$

Invariance under the shift symmetry

$$
A \rightarrow A+a_{1}(t-z), \quad A^{\dagger} \rightarrow A^{\dagger}+a_{2}(t-z)
$$

requires $Z_{A}$ to be 1 . 


\section{THE FULL MODEL AND ITS GEOMETRY}

The complex Grassmannian manifold is a manifold consisting of all $M$-dimensional subspaces of an $(M+N)$ dimensional complex vector space. Since a subspace is uniquely determined by its complement, it can also be treated as a manifold of $N$-dimensional subspaces, which makes $\mathcal{G}_{M, N}$ and $\mathcal{G}_{M, N}$ equivalent. The Grassmannian is a homogenous space, i.e., a space that can be represented as a quotient of a group, acting transitively on a manifold, over the stabilizer of a certain element. In the present case, $\mathbb{S U}(N+M)$ acts on $\mathbb{C}^{N+M}$ whose $M$-dimensional subspace is invariant under its rotations, given by $\mathbb{S U}(M)$, and rotations of the complement, given by $\mathbb{S U}(N)$, which justifies the definition (1). The complex Grassmannian admits a Kähler-Einstein metric - the one that is both Kählerian [i.e., is defined by a single real closed $(1,1)$ form, the Kähler potential, see Eq. (24) below] and is proportional to the Ricci tensor,

$$
R_{i \bar{j}}=b \frac{g^{2}}{2} G_{i \bar{j}}
$$

where $g$ is the coupling constant and $b$ is the dual Coxeter number.

The complex dimension of the Grassmannian manifold is $M N$. In terms of the $M N$ chiral superfields, labeled by two indices, that were introduced above,

$$
\begin{aligned}
\Phi^{i \alpha} & =\phi^{i \alpha}+\sqrt{2} \theta \psi^{i \alpha}+\theta^{2} F^{i \alpha}, \quad n=1 \ldots N, \\
\alpha & =1 \ldots M,
\end{aligned}
$$

a generic undeformed $\mathcal{N}=(2,2)$ model the Lagrangian can be written as

$$
\mathcal{L}_{(2,2)}=\int d^{4} \theta K\left(\Phi, \Phi^{\dagger}\right)
$$

where $K\left(\Phi, \Phi^{\dagger}\right)$ is the Kähler potential, which depends on the chiral and antichiral fields.

Since interactions in the undeformed model are caused by geometry, we now review some details about geometric structure of the target space. The Kähler potential of the Grassmannian manifold is given by

$$
K=\frac{2}{g^{2}} \operatorname{Tr} \ln \left(\delta^{n \bar{m}}+\Phi^{n \gamma} \Phi^{\dagger \bar{\gamma} \bar{m}}\right),
$$

where the trace is taken over the latin indices. Obviously, we could also define $K=\operatorname{Tr} \ln \left(\delta^{\alpha \bar{\beta}}+\Phi^{n \alpha} \Phi^{\dagger \bar{\beta} \bar{n}}\right)$ and take the trace with respect to the greek indices. The subscripts are reserved for lowering with the aid of the metric tensor. The first derivatives of the Kähler potential have the form of

$$
\begin{aligned}
K^{\bar{\beta} \bar{j}} & \equiv \frac{\partial}{\partial \Phi^{\dagger \bar{\beta} \bar{j}}} K=\frac{2}{g^{2}} \Phi^{n \beta}\left[\left(\hat{\mathbb{1}}_{N}+\Phi \Phi^{\dagger}\right)^{-1}\right]^{\bar{j} n}, \\
\bar{K}^{\alpha i} & \equiv \frac{\partial}{\partial \Phi^{\alpha i}} K=\frac{2}{g^{2}} \Phi^{\dagger \bar{n} \bar{\alpha}}\left[\left(\hat{\mathbb{1}}_{N}+\Phi \Phi^{\dagger}\right)^{-1}\right]^{\bar{i} n} .
\end{aligned}
$$

The Kähler metric is obtained as follows:

$$
\begin{aligned}
G_{i \bar{j} \alpha \bar{\beta}}= & \frac{\partial}{\partial \Phi^{i \alpha}} \frac{\partial}{\partial \Phi^{\dagger \bar{\beta} \bar{j}}} K=\frac{2}{g^{2}} \operatorname{Tr}\left\{\delta^{n i} \delta^{\alpha \bar{\beta}}\left[\left(\hat{\mathbb{1}}_{N}+\Phi \Phi^{\dagger}\right)^{-1}\right]^{\bar{j} m}\right. \\
& \left.-\Phi^{n \beta}\left[\left(\hat{\mathbb{1}}_{N}+\Phi \Phi^{\dagger}\right)^{-1}\right]^{\bar{j} i} \Phi^{\dagger \bar{\alpha} \bar{l}}\left[\left(\hat{\mathbb{1}}_{N}+\Phi \Phi^{\dagger}\right)^{-1}\right]^{\bar{l} m}\right\} \\
= & \frac{2}{g^{2}}\left[\left(\hat{\mathbb{1}}_{N}+\Phi \Phi^{\dagger}\right)^{-1}\right]^{\bar{i} j}\left[\left(\hat{\mathbb{1}}_{M}+\Phi \Phi^{\dagger}\right)^{-1}\right]^{\bar{\alpha} \beta} .
\end{aligned}
$$

The small- $\Phi$ expansion of the metric, which is to be used in the background-field method, has the form

$G_{i j \alpha \bar{\beta}}=\frac{2}{g^{2}}\left[\delta^{i \bar{j}} \delta^{\alpha \bar{\beta}}-\delta^{\alpha \bar{\beta}} \Phi^{j \gamma} \Phi^{\dagger \bar{\gamma} \bar{i}}-\delta^{i \bar{j}} \Phi^{n \beta} \Phi^{\dagger \bar{\alpha} n}\right]+\ldots$

where the dots stand for the higher-order terms.

The Ricci tensor is proportional to the metric,

$$
R_{i \bar{j} \alpha \bar{\beta}}=\frac{g^{2}}{2}(M+N) G_{i \bar{j} \alpha \bar{\beta}},
$$

which is a particular case of (21). Further details can be found in a review paper [40], Sec. IV B.

We are now in a position to apply these results to the full model. which combines the geometric structure with the partial supersymmetry breaking. To break supersymmetry, we add another term to the Lagrangian, which is similar to the one in the previous section.

Since $\mathcal{B}$ is a singlet with respect to the isometry group, its introduction does not affect the geometry of the model. Thus, we get the following expression for the Lagrangian of the full model in the $(0,2)$ formalism:

$$
\begin{aligned}
\mathcal{L}_{\mathcal{G}_{M, N}}= & \frac{1}{4} d^{2} \theta\left[\bar{K}_{\alpha i}\left(A, A^{\dagger}\right)\left(i \partial_{R} A^{\alpha i}-2 \kappa \mathcal{B} B^{\alpha i}\right)+\text { H.c. }\right] \\
& +\frac{1}{2} \int d^{2} \theta\left[Z_{B} G_{i \bar{j} \alpha \bar{\beta}}\left(A, A^{\dagger}\right) B^{\dagger \bar{\beta} \bar{j}} B^{\alpha i}+Z_{\mathcal{B}} \mathcal{B}^{\dagger} \mathcal{B}\right] .
\end{aligned}
$$

Next, we proceed to finding the beta functions of this model.

\section{BETA FUNCTIONS OF THE FULL MODEL}

In components, the Lagrangian of the full model (29) reads as 


$$
\begin{aligned}
& \mathcal{L}_{\mathcal{G}_{M, N}}=G_{i \bar{j} \alpha \bar{\beta}}\left[\partial_{L} \phi^{\dagger \bar{j} \bar{\beta}} \partial_{R} \phi^{\alpha i}+\psi_{L}^{\dagger j \bar{j} \bar{\beta}} i \nabla_{R} \psi_{L}^{\alpha i}+Z_{B} \psi_{R}^{\dagger j \bar{\beta}} i \nabla_{L} \psi_{R}^{\alpha i}\right] \\
& +Z_{B} R_{i \bar{j} k \bar{l} \alpha \bar{\beta} \gamma \bar{\delta}} \psi_{L}^{\dagger \bar{j} \bar{\beta}} \psi_{L}^{\alpha i} \psi_{R}^{\dagger \bar{\dagger} \bar{\delta}} \psi_{R}^{\gamma k}+Z_{\mathcal{B}} \zeta_{R}^{\dagger} i \partial_{L} \zeta_{R} \\
& +\left[\kappa \zeta_{R} G_{i \bar{j} \alpha \bar{\beta}}\left(i \partial_{L} \phi^{\dagger \bar{j} \bar{\beta}} \psi_{R}^{\alpha i}\right)+\text { H.c. }\right] \\
& +\frac{|\kappa|^{2}}{Z_{B}} \zeta_{R}^{\dagger} \zeta_{R}\left(G_{i \bar{j} \alpha \bar{\beta}} \psi_{L}^{\dagger \bar{j} \bar{\beta}} \psi_{L}^{\alpha i}\right) \\
& -\frac{|\kappa|^{2}}{Z_{\mathcal{B}}}\left(G_{i \bar{j} \alpha \bar{\beta}} \psi_{L}^{\dagger \bar{j} \bar{\beta}} \psi_{R}^{\alpha i}\right)\left(G_{k \bar{\gamma} \gamma \bar{\delta}} \psi_{R}^{\dagger \dagger \bar{l}{ }^{\gamma}} \psi_{L}^{\gamma k}\right) .
\end{aligned}
$$

Here $\kappa$ is the heterotic deformation parameter. At $\kappa=0$, the field $\mathcal{B}$ becomes sterile, and the $(2,2)$ supersymmetry is restored for other fields $\left(Z_{B}\right.$ is not running at $\kappa=0$, and can be taken to be 1).

In the $\kappa^{2} / g^{2} \rightarrow \infty$ limit, the theory reduces to the linearized model (9). The constants $\kappa$ and $\gamma$ are related as

$$
\gamma^{2}=\frac{\kappa^{2}}{Z_{B} Z_{\mathcal{B}}}
$$

Note that the Lagrangian (29) does not contain $Z_{A}$, which can be absorbed in $g$. Thus, the bare parameters of the model are $g, \gamma, Z_{B}$, and $Z_{\mathcal{B}}$. Their renormalization can be calculated either using the superfield formalism, as was done in [18], or by means of the background-field method, which we briefly review in Appendix B.

Extending the analysis to the two-loop level (see Figs. 2, 3,4 for the corresponding diagrams) gives

$$
\begin{aligned}
\beta\left(g^{2}\right)_{\text {two-loop }}= & -\frac{g^{2}}{4 \pi}\left[g^{2}(M+N)\left(1+\frac{\gamma^{2}}{2 \pi}\right)\right. \\
& \left.-(M N+1) \frac{\gamma^{4}}{2 \pi}\right], \\
\beta\left(\gamma^{2}\right)_{\text {two-loop }}= & -\frac{\gamma^{2} /(2 \pi)}{1-\left(\gamma^{2} / 4 \pi\right)}\left[(M+N) g^{2}-(M N+1) \gamma^{2}\right. \\
+ & \left.\frac{\gamma^{2}}{8 \pi}\left((M+N) g^{2}-2(M N+1) \gamma^{2}\right)\right] .
\end{aligned}
$$

As expected, Eqs. (32) and (33) reproduce the results for the $\mathbb{C P}(N-1)$ model as one substitutes $\{M, N\} \rightarrow$ $\{1, N-1\}$ [24].

\section{LARGE- $N$ LIMIT}

We now briefly discuss what we can learn from the results above regarding the large- $N$ expansion of this theory.

From (32) one can immediately read off the 't Hooft large- $N$ expansion parameter

$$
t \equiv g^{2}(M+N)
$$

There are a number of limits one can study: (a) $M$ fixed; $N \rightarrow \infty$.

(b) $\nu=M / N$ fixed, $\nu \ll 1 ; N \rightarrow \infty$.

(c) $\nu=M / N$ fixed, $\nu \sim 1 ; N \rightarrow \infty$.

The second case is somewhat analogous to the Veneziano limit it QCD [43]. In such a setting, a larger number of planar diagrams, as compared to the case of ordinary large $N$, survives. By examining (32) and (33), we deduce that, in order to have a sensible limit, the second expansion parameter has to be defined as

$$
\tilde{t} \equiv \gamma^{2} M N
$$

and be finite. Qualitatively, we can understand from the $\mathcal{B}$ one-loop propagator that has no external loops and the $M N$ degrees of freedom (indices) running in the loop and $\gamma$ at each of the vertices. The limit above makes such a diagram finite.

In this case, the beta functions can be defined purely in terms of the t' Hooft parameters,

$$
\begin{gathered}
\beta(t)_{\mathrm{two}-\mathrm{loop}}=-\frac{t^{2}}{4 \pi}, \\
\beta(\tilde{t})_{\mathrm{two-loop}}=-\frac{\tilde{t}}{2 \pi}(t-\tilde{t}) .
\end{gathered}
$$

The higher-order corrections vanish in this approximation, as it can be seen from (32) and (33), and the expressions above reduce to one loop. It should be noted that the 't Hooft limit here is defined at UV. In IR the coupling constants grow while $M$ and $N$ remain the same, so the limit is not valid anymore.

What are the other consequences of considering such a limit? Let us take a look at the fixed-point behavior of the theory. The ratio $\frac{\gamma}{g^{2}}$, being a constant in the superpotential, is not getting renormalized. However, one may be interested in the ratio

$$
\rho \equiv \frac{\gamma^{2}}{g^{2}}
$$

appearing in front of the four-fermion interaction. The corresponding one-loop beta function acquires the form of

$$
\beta(\rho)=\frac{\rho g^{2}}{4 \pi}[2(M N+1) \rho-(M+N)] .
$$

It has a fixed point

$$
\rho_{*}=\frac{1}{2} \frac{M+N}{M N+1} .
$$

Its behavior as a function of $M$ and $N$ is illustrated in Fig 5 . The fixed point (40) remains at two loops; the appropriate numerical result is shown in Fig. 6. For $M$ being constant, this fixed point approaches the asymptotic value 


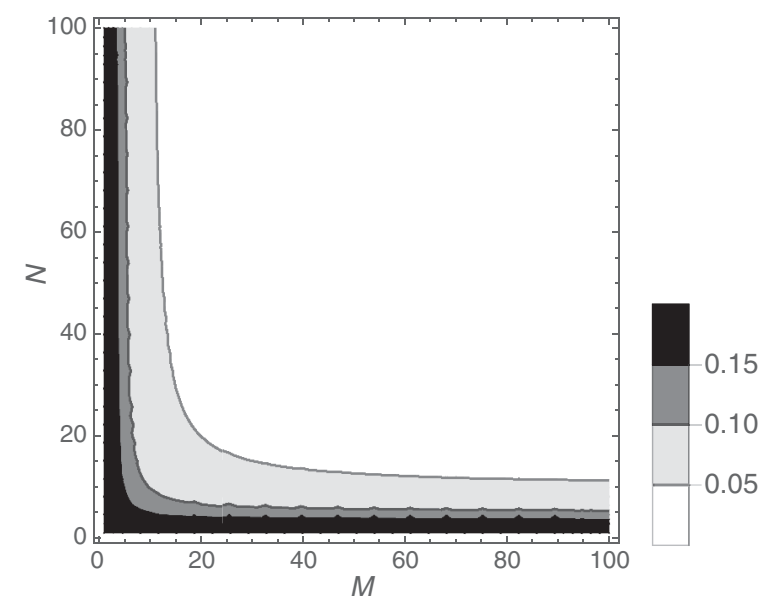

FIG. 5. Fixed point $\rho_{*}$ for the ratio $\rho \equiv \gamma^{2} / g^{2}$ as a function of $M$ and $N$. The value of $\rho_{*}$ is denoted by brightness (the scale is on the right) while $M$ and $N$ run along both axes. We see that if one of the parameters scales with the other one, $\rho_{*}$ approaches 0 no matter what that ratio is.

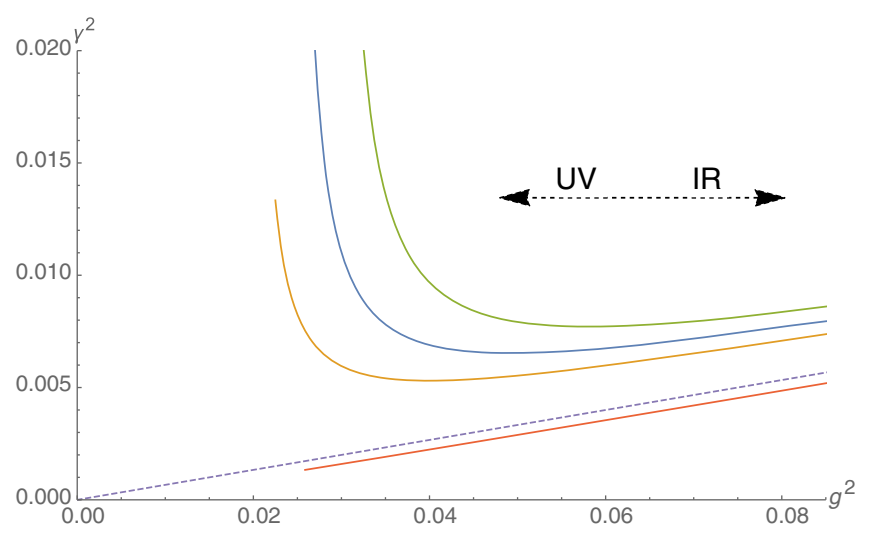

FIG. 6. The renormalization group flow of the coupling constants $g^{2}$ and $\gamma^{2}$, based on the two-loop expressions (32) and (33). Here $M=10, N=30$. The dashed line has the slope $\rho_{*}=\frac{M+N}{2(M N+1)}$, so the curves above and below correspond to $\rho$ being greater and smaller than $\rho_{*}$, respectively. We see that the coupling constants flow to that ratio at the IR.

$$
\rho_{*} \rightarrow \frac{1}{2 M} \quad(N \rightarrow \infty, M \text { fixed }) .
$$

For $M=1$ one recovers the result $\rho_{*}=1 / 2$ for the $\mathbb{C P}(N)$ model [44]. By looking at (40) we conclude that, if $M$ scales with $N$, the fixed-point value becomes

$$
\rho_{*} \rightarrow 0 \quad\left(N \rightarrow \infty, \varkappa \equiv \frac{M}{N}=\text { const } \neq 0\right),
$$

which is the case for the Veneziano limit. Interestingly, we obtain that the value for the fixed point does not depend on $\varkappa$ once $M$ scales with $N$. There is no difference between the
Veneziano limit of a small but finite $\varkappa$ and the case when $M \sim N$ and $\varkappa \sim 1$.

From (39) we see that for consistency we have to choose

$$
\rho \leq \rho_{*}
$$

in order to avoid the Landau pole. In the opposite case, one faces the situation similar to that in the linearized model [18].

\section{CONCLUSIONS}

In this paper, we have discussed the perturbative aspects of the heterotically deformed $(0,2)$ Grassmannian $\mathcal{G}_{M, N}$ model, as well as of its linearized version. In the latter case, the only coupling constant is the deformation parameter, whose running is determined by the renormalization of the fields solely - which can be proved nonperturbatively. The beta function stays positive, and the theory possesses a Landau pole in UV.

A similar kind of behavior takes place in the full model-however, only for a certain range of parameters. There, in contradistinction with the linearized model, for a different set of parameters one may also end up with a welldefined asymptotically free theory, reaching its conformal point at the UV.

Lastly, we have discussed the large- $N$ and Veneziano limits of the model, which will be subject to more detailed research in the upcoming publication [45]. We found that the large- $N$ behavior of the coupling's fixed-point value depends crucially on the type of limit used.

\section{ACKNOWLEDGMENTS}

The authors are grateful to J. Chen both for the stimulating exchange that gave a start to this work and for the advise provided at its later stages. The authors also thank X. Cui for useful discussions on the topics raised in this paper. The work of M. S. was supported in part by the DOE Grant No. DE-SC0011842. E. K. was supported in part by the Robert E Greiling Jr. Scholarship for Particle Physics and Cosmic Rays. M. K. was supported by DOE HEP Award No. 0000240346.

\section{APPENDIX A: CONVENTIONS}

Gamma matrices, metric, $\varepsilon$-symbols:

$$
\begin{aligned}
& \gamma^{0}=\sigma_{2}=\left(\begin{array}{cc}
0 & -i \\
i & 0
\end{array}\right), \quad \gamma^{1}=i \sigma_{1}=\left(\begin{array}{ll}
0 & i \\
i & 0
\end{array}\right), \\
& \gamma^{5}=\gamma^{0} \gamma^{1}=\sigma^{3}=\left(\begin{array}{cc}
1 & 0 \\
0 & -1
\end{array}\right) . \\
& \epsilon_{\alpha \beta}=i \sigma_{2}=\left(\begin{array}{cc}
0 & 1 \\
-1 & 0
\end{array}\right), \quad \epsilon^{\alpha \beta}=i \sigma_{2}=\left(\begin{array}{cc}
0 & -1 \\
1 & 0
\end{array}\right) .
\end{aligned}
$$




$$
\begin{gathered}
g^{\mu \nu}=\left(\begin{array}{cc}
1 & 0 \\
0 & -1
\end{array}\right), \quad g_{\mu \nu}=\left(\begin{array}{cc}
1 & 0 \\
0 & -1
\end{array}\right) . \\
\gamma^{\mu} \gamma^{\nu}+\gamma^{\nu} \gamma^{\mu}=2 g^{\mu \nu} . \\
\alpha, \beta=1,2 \equiv R, L, \quad \mu, \nu=0,1=t, z .
\end{gathered}
$$

Spinors:

$$
\psi=\left(\begin{array}{c}
\psi_{R} \\
\psi_{L}
\end{array}\right), \quad \psi^{\dagger}=\left(\begin{array}{ll}
\psi_{R}^{\dagger} & \psi_{L}^{\dagger}
\end{array}\right), \quad \bar{\psi}=\psi^{\dagger} \gamma^{0}
$$

Light cone coordinates:

$$
\begin{array}{ll}
x^{L}=\frac{1}{2}\left(x^{0}+x^{1}\right), & \partial_{R}=\partial_{t}-\partial_{z}, \\
x^{R}=\frac{1}{2}\left(x^{0}-x^{1}\right), & \partial_{L}=\partial_{t}+\partial_{z} .
\end{array}
$$

Supercharges:

$$
\begin{aligned}
& \left\{\mathcal{Q}_{R}, \overline{\mathcal{Q}}_{R}\right\}=-2 i \partial_{R}=-2(\mathcal{H}+\mathcal{P}) \\
& \left\{\mathcal{Q}_{L}, \overline{\mathcal{Q}}_{L}\right\}=-2 i \partial_{L}=-2(\mathcal{H}-\mathcal{P})
\end{aligned}
$$

Spinor contraction:

$$
\begin{gathered}
\psi \theta=\psi^{\alpha} \theta_{\alpha}=\psi^{\alpha} \epsilon_{\alpha \beta} \theta^{\beta}=\psi_{R} \theta_{L}-\psi_{L} \theta_{R}, \\
\bar{\psi} \theta=\bar{\psi} \hat{\mathbb{1}} \theta=i\left(\psi_{L}^{\dagger} \theta_{R}-\psi_{R}^{\dagger} \theta_{L}\right), \\
\bar{\psi} \gamma^{5} \theta=i\left(\psi_{L}^{\dagger} \theta_{R}+\psi_{R}^{\dagger} \theta_{L}\right), \\
\bar{\psi} \gamma^{0} \theta=i\left(\psi_{R}^{\dagger} \theta_{R}+\psi_{L}^{\dagger} \theta_{L}\right), \quad \bar{\psi} \gamma^{1} \theta=i\left(\psi_{R}^{\dagger} \theta_{R}-\psi_{L}^{\dagger} \theta_{L}\right) .
\end{gathered}
$$

Integration:

$$
(\bar{\theta} \theta)=i\left(\theta_{L}^{\dagger} \theta_{R}-\theta_{R}^{\dagger} \theta_{L}\right), \quad(\bar{\theta} \theta)(\bar{\theta} \theta)=-2 \theta_{R} \theta_{L} \theta_{R}^{\dagger} \theta_{L}^{\dagger}
$$

$$
\begin{gathered}
\int d \theta_{R} \theta_{R}=1, \quad \int d \theta_{L} \theta_{L}=1, \quad \int d \theta_{R}^{\dagger} \theta_{R}^{\dagger}=1, \\
\int d \theta_{R}^{\dagger} \theta_{R}^{\dagger}=1, \\
\int d \theta_{R} d \theta_{L}^{\dagger}(\bar{\theta} \theta)=i, \quad \int d \theta_{L} d \theta_{R}^{\dagger}(\bar{\theta} \theta)=i, \quad(\mathrm{~A} 11 \mathrm{~B}) \\
\int d \theta_{R} d \theta_{L} d \theta_{R}^{\dagger} d \theta_{L}^{\dagger}(\bar{\theta} \theta)(\bar{\theta} \theta)=2 .
\end{gathered}
$$

Chiral coordinates:

$$
y^{\mu}=x^{\mu}+\bar{\theta} \gamma^{\mu} \theta
$$

\section{APPENDIX B: BACKGROUND-FIELD METHOD}

Following the lines for $\mathbb{C P}(N-1)[17,46]$, we start with calculating the beta function for the Grasmannian model. The beta function can be read off from the renormalization of the coupling constant, which we calculate using the background-field method.

We begin with splitting the quantum field $\phi(x)$ in two parts, ${ }^{2}$

$$
\phi(x)=\phi_{0}(x)+q(x)
$$

Here $\phi_{0}(x)$ denotes the background field, which can be chosen arbitrarily, while $q(x)$ is the quantum correction to it. $^{3}$

We then can calculate the renormalized coupling constant by integrating out the quantum corrections to the field configuration $\phi_{0}(x)$,

$$
\begin{aligned}
& \exp \left\{-\int d^{2} x \mathcal{L}\left[g_{r}, \phi_{r}(x)\right]\right\} \\
& \equiv \int \mathcal{D} \phi \exp \left\{-\int d^{2} x \mathcal{L}\left[g_{b}, \phi(x)\right]\right\} \\
& =\int \mathcal{D} q \exp \left\{-\int d^{2} x \mathcal{L}\left[g_{b}, \phi_{0}(x)+q(x)\right]\right\},
\end{aligned}
$$

where $g_{b}$ and $g_{r}$ denote the bare and renormalized couplings, correspondingly. The rhs of (B2) is calculated by expanding the exponent into the series in $q(x)$. As we see shortly, once the background field $\phi_{0}(x)$ has been chosen in a convenient way, the expansion of the Lagrangian acquires the form of

$$
\begin{aligned}
9 \mathcal{L}\left[g_{b}, \phi_{0}+q\right]= & \mathcal{L}\left[g_{b}, \phi_{0}\right]+q \times(\ldots) \\
& +C\left[\phi_{0}\right](\partial q)^{2}+V(q, \partial q) .
\end{aligned}
$$

The first term is the background Lagrangian to which the quantum corrections are to be calculated. The term linear in $q$ has to vanish within the background field method. This either happens automatically when $\phi_{0}$ obeys the classical equations of motion, or is otherwise achieved by adding the

\footnotetext{
${ }^{2}$ In Eqs. (B1)-(B3) it is implied that all possible indices are suppressed/contracted.

${ }^{3}$ Strictly speaking, the presented approach is noncovariant from the target-space point of view. Under the assumption that both $\phi(x)$ and $\phi_{0}(x)$ in (B1) belong to the target space, their difference $q(x)$ is not a well-defined geometric structure. A more careful treatment can be found in [47], where $q(x)$ is replaced by $\xi(x)$, a unit tangent vector along the target-space geodesic connecting $\phi(x)$ and $\phi_{0}(x)$.
} 
appropriate source terms. The third term defines the free propagator of the field $q\left(C\left[\phi_{0}\right]\right.$ is the quadratic coefficient in the Taylor expansion of $\mathcal{L}\left[g_{b}, \phi_{0}+q\right]$ ). By calculating the loop corrections to it, we obtain the wave function renormalization. Lastly, by $V(q, \partial q)$ we have denoted all the remaining terms in the expansion of $\mathcal{L}\left[g_{b}, \phi_{0}+q\right]$. Those contain an infinite number of terms that can be represented by diagrams with $\phi_{0}$-dependent vertices.

For definiteness, we present the calculation of the oneloop beta function of the bosonic Grassmannian model. Following the steps from Sec. 28 of [46], we obtain, in analogy with (28.29) in ibid.,

$\mathcal{L}^{[2]}=2 \partial_{\mu} \bar{q}^{n \alpha} \partial^{\mu} q^{n \alpha}-2 k^{2}|f|^{2}\left(\sum_{n=1}^{N} \bar{q}^{n 1} q^{n 1}+\sum_{\alpha=1}^{M} \bar{q}^{1 \alpha} q^{1 \alpha}\right)$.

Comparing the equation above with the $\mathbb{C P}(N-1)$ case, we deduce that ${ }^{4}$

$$
\beta\left(g^{2}\right)_{\text {one-loop }}=-\frac{g^{4}(M+N)}{4 \pi},
$$

which matches the result of [38]. We recognize the dual Coxeter number $T_{S U(M+N)}=M+N$, which also appears in the metric, and in the Ricci tensor.

Next, we follow the steps of [17] and calculate the $Z$-factors for the heterotically deformed $(0,2)$ model. We keep using the background-field method and perform the

${ }^{4} \mathbb{C P}(N-1)=\mathcal{G}_{N-1,1}$. calculations in components. The only vertex relevant for the one-loop calculation is $\left(\gamma \zeta_{R} G_{i \bar{j} \alpha \bar{\beta}}\left(i \partial_{L} \phi^{\dagger \bar{\beta} \bar{j}}\right) \psi_{R}^{i \alpha}+\right.$ H.c. $)$.

To the lowest order, the diagram for the wave function renormalization takes the form

$$
\begin{aligned}
& \gamma^{2} \zeta_{R}(x) \delta^{i \bar{j}} \delta^{\alpha \bar{\beta}} \delta^{k \bar{l}} \delta^{\gamma \bar{\delta}} \psi_{R}^{i \alpha}(x)\left(i \partial_{L} \bar{q}^{\bar{\beta} \bar{j}}(x)\right) \\
& \times\left(-i \partial_{L} q^{k \gamma}(y)\right) \bar{\psi}_{R}^{\bar{\delta} \bar{l}}(y) \zeta_{R}^{\dagger}(y) .
\end{aligned}
$$

For each of $\psi_{R}^{i \alpha}(x)$, the diagram is identical to the $\mathbb{C P}(1)$ case, while for $\zeta_{R}$ we are getting an additional $M N$ factor corresponding to the number of fields in the loop.

This way, for the $\psi_{R}^{i \alpha}(x)$ field one gets

$$
Z_{\psi}=1+i \gamma^{2} I,
$$

while for $\zeta_{R}$

$$
Z_{\zeta}=1+M N \gamma^{2} I
$$

Here

$$
I=\int \frac{d^{d} p}{(2 \pi)^{d}} \frac{1}{p^{2}-\mu^{2}}=\frac{1}{2 \pi} \log \left(\frac{M_{\mathrm{uv}}}{\mu}\right) .
$$

To the first order, the bosonic beta function (B5) remains intact, while the beta function for the deformation parameter is

$$
\beta(\gamma)_{\text {one-loop }}=\frac{\gamma}{4 \pi}\left[(M+N) g^{2}-(M N+1) \gamma^{2}\right] .
$$

To proceed further, we take into account the diagrams contributing to the renormalization of $B$ and $\mathcal{B}$ at two loops.
[1] M. Gell-Mann and M. Levy, The axial vector current in beta decay, Nuovo Cimento 16, 705 (1960).

[2] A. M. Polyakov, Interaction of goldstone particles in twodimensions. Applications to ferromagnets and massive Yang-Mills fields, Phys. Lett. 59B, 79 (1975).

[3] M. Shifman and A. Yung, Heterotic flux tubes in $N=2$ SQCD with $N=1$ preserving deformations, Phys. Rev. D 77, 125016 (2008); Erratum 79, 049901 (2009).

[4] K. Intriligator, Fusion residues, Mod. Phys. Lett. A 06, 3543 (1991).

[5] C. Vafa, Topological mirrors and quantum rings, edited by S. T. Yau, Mirror symmetry I (American Mathematical Society, Providence, RI and International Press, Cambridge, MA, 1998), p. 97-120.

[6] E. Witten, The verlinde algebra and the cohomology of the grassmannian, in Geometry, topology, and physics. Proceedings, Conference dedicated to Raoul Bott on his 70th Birthday, Cambridge, USA, 1993, edited by Y. ShingTung (International Press, Cambridge, MA, 1995), p. 357, https://www.worldcat.org/title/geometry-topology-physicsfor-raoul-bott/oclc/33865839.

[7] M. Bourdeau, Grassmannian sigma models and topological-antitopological fusion, Nucl. Phys. B439, 421 (1995).

[8] S. Stolz, A conjecture concerning positive Ricci curvature and the Witten genus, Math. Ann. 304, 785 (1996).

[9] D. Bak, S.-O. Hahn, J. Lee, and P. Oh, Supersymmetric Q-lumps in the Grassmannian nonlinear sigma models, Phys. Rev. D 75, 025004 (2007).

[10] A. Adams, A. Basu, and S. Sethi, $(0,2)$ duality, Adv. Theor. Math. Phys. 7, 865 (2003).

[11] E. Witten, Two-dimensional models with $(0,2)$ supersymmetry: Perturbative aspects, Adv. Theor. Math. Phys. 11, 1 (2007). 
[12] M. Shifman and A. Yung, Large- $N$ solution of the heterotic $N=(0,2)$ two-dimensional $\mathrm{CP}(\mathrm{N}-1)$ model, Phys. Rev. D 77, 125017 (2008); Erratum 81, 089906 (2010).

[13] M.-C. Tan and J. Yagi, Chiral algebras of $(0,2)$ sigma models: Beyond perturbation theory, Lett. Math. Phys. 84, 257 (2008).

[14] J. Yagi, Chiral algebras of $(0,2)$ models, Adv. Theor. Math. Phys. 16, 1 (2012).

[15] P. Koroteev and A. Monin, Large- $N$ solution of the heterotic $N=(0,1)$ two-dimensional $O(N)$ sigma model, Phys. Rev. D 81, 105001 (2010).

[16] P. Koroteev, A. Monin, and W. Vinci, Large-N solution of the heterotic weighted nonlinear sigma-model, Phys. Rev. D 82, 125023 (2010).

[17] X. Cui and M. Shifman, Perturbative aspects of heterotically deformed CP(N-1) sigma model. I, Phys. Rev. D 82, 105022 (2010).

[18] X. Cui and M. Shifman, $N=(0,2)$ Supersymmetry and a nonrenormalization theorem, Phys. Rev. D 84, 105016 (2011).

[19] X. Cui and M. Shifman, $N=(0,2)$ deformation of $\mathrm{CP}(1)$ model: Two-dimensional analog of $N=1$ Yang-Mills theory in four dimensions, Phys. Rev. D 85, 045004 (2012).

[20] I. Melnikov, S. Sethi, and E. Sharpe, Recent developments in $(0,2)$ mirror symmetry, SIGMA 8, 068 (2012).

[21] A. Gadde, S. Gukov, and P. Putrov, $(0,2)$ trialities, J. High Energy Phys. 03 (2014) 076.

[22] A. Gadde, S. Gukov, and P. Putrov, Exact Solutions of 2d Supersymmetric Gauge Theories, arXiv:1404.5314.

[23] B. Jia, E. Sharpe, and R. Wu, Notes on nonabelian $(0,2)$ theories and dualities, J. High Energy Phys. 08 (2014) 017.

[24] J. Chen, X. Cui, M. Shifman, and A. Vainshtein, $N=(0,2)$ deformation of $(2,2)$ sigma models: Geometric structure, holomorphic anomaly, and exact $\beta$ functions, Phys. Rev. D 90, 045014 (2014).

[25] A. J. Peterson, E. Kurianovych, and M. Shifman, More on two-dimensional $O(N)$ models with $\mathcal{N}=(0,1)$ supersymmetry, Phys. Rev. D 93, 065016 (2016).

[26] J. Chen, X. Cui, M. Shifman, and A. Vainshtein, Anomalies of minimal $\mathcal{N}=(0,1)$ and $\mathcal{N}=(0,2)$ sigma models on homogeneous spaces, J. Phys. A 50, 025401 (2017).

[27] J. Chen, X. Cui, M. Shifman, and A. Vainshtein, On isometry anomalies in minimal $N=(0,1)$ and $N=(0,2)$ sigma models, Int. J. Mod. Phys. A 31, 1650147 (2016).

[28] G. V. Dunne and Mithat Unsal, Resurgence and dynamics of $\mathrm{O}(\mathrm{N})$ and Grassmannian sigma models, J. High Energy Phys. 09 (2015) 199.

[29] M. Shifman and A. Yung, Two-dimensional sigma models related to nonAbelian strings in super-Yang-Mills, in Pomeranchuk 100, Proceedings Alikhanov Institute of
Theoretical and Experimental Physics (ITEP), Moscow, 2014, edited by A. Gorsky and M. Vysotsky(2014), p. 181, https://doi.org/10.1142/9789814616850_0011.

[30] H. Eichenherr, SU(N) invariant nonlinear sigma models, Nucl. Phys. B146, 215 (1978); Nucl. Phys. B155, 544 (1979).

[31] V. L. Golo and A. M. Perelomov, A few remarks on chiral theories with sophisticated topology, Lett. Math. Phys. 2, 477 (1978).

[32] E. Cremmer and J. Scherk, The supersymmetric nonlinear sigma model in four-dimensions and its coupling to supergravity, Phys. Lett. 74B, 341 (1978).

[33] E. Witten, A supersymmetric form of the nonlinear sigma model in two-dimensions, Phys. Rev. D 16, 2991 (1977).

[34] P. Di Vecchia and S. Ferrara, Classical solutions in twodimensional supersymmetric field theories, Nucl. Phys. B130, 93 (1977).

[35] V. A. Novikov, M. A. Shifman, A. I. Vainshtein, and V. I. Zakharov, Two-dimensional sigma models: Modeling nonperturbative effects of quantum chromodynamics, Fiz. Elem. Chastits At. Yadra 17, 472 (1986); [Phys. Rep. 116, 103 (1984)].

[36] R. D. Pisarski, Nonlinear $\sigma$ models of symmetric spaces, Phys. Rev. D 20, 3358 (1979).

[37] J. W. van Holten, Structure of Grassmannian $\sigma$ models, Z. Phys. C 27, 57 (1985).

[38] A. Y. Morozov, A. M. Perelomov, and M. A. Shifman, Exact Gell-Mann-Low function of supersymmetric Kähler sigma models, Nucl. Phys. B248, 279 (1984).

[39] E. Abdalla, M. Forger, and A. L. Santos, Nonlocal charges for nonlinear $\sigma$ models on Grassmann manifolds, Nucl. Phys. B256, 145 (1985).

[40] A. M. Perelomov, Chiral models: Geometrical aspects, Phys. Rep. 146, 135 (1987).

[41] A. M. Perelomov, Supersymmetric chiral models: Geometrical aspects, Phys. Rep. 174, 229 (1989).

[42] N. Seiberg, Naturalness versus supersymmetric nonrenormalization theorems, Phys. Lett. B 318, 469 (1993).

[43] G. Veneziano, Some aspects of a unified approach to gauge, dual and Gribov theories, Nucl. Phys. B117, 519 (1976).

[44] X. Cui, Perturbative and nonperturbative aspects of heterotic sigma models, Ph.D. thesis, University of Minnesota, 2012.

[45] M. Kreshchuk, E. Kurianovych, and M. Shifman (to be published).

[46] M. Shifman, Advanced Topics in Quantum Field Theory: A Lecture Course (Cambridge University Press, Cambridge, England, 2012).

[47] S. V. Ketov, Quantum Nonlinear Sigma Models: From Quantum Field Theory to Supersymmetry, Conformal Field Theory, Black Holes and Strings (Springer, New York, 2000). 